\title{
The role of schools in driving SARS-CoV-2 transmission: not just an open-and-shut case
}

\author{
Laura F White $^{1^{*}}$, Eleanor J Murray², Arijit Chakravarty ${ }^{3}$ \\ ${ }^{1}$ Department of Biostatistics, Boston University School of Public Health, Boston MA \\ ${ }^{2}$ Department of Epidemiology, Boston University School of Public Health, Boston MA \\ ${ }^{3}$ Fractal Therapeutics, Cambridge MA \\ * To whom correspondence should be addressed: Ifwhite@bu.edu
}

\begin{abstract}
Summary
Keeping schools open without permitting COVID-19 spread has been complicated by conflicting messages around the role of children and schools in fueling the pandemic. Here, we describe methodological limitations of research minimizing SARS-CoV-2 transmission in schools, and we review evidence for safely operating schools while reducing overall SARS-CoV-2 transmission.
\end{abstract}

\section{Main Text}

Schools are a critical part of any society in training the next generation, providing important socialization, and enabling the economy to function. They are also recognized drivers of infectious disease spread. We provide evidence that SARS-CoV-2 can be transmitted in schools, and that this affects not only those in schools, but also the communities inextricably linked with them. Therefore, when school conditions favor rapid spread of SARS-CoV-2, this impacts the course of the pandemic. Importantly, in the absence of systematic and widespread surveillance testing, it is challenging to detect transmission between schools and the community. ${ }^{1}$ Rampant transmission fuels viral evolution with the emergence of new viral strains that gain competitive advantage by evading prior immunity, with no promises that they will be less virulent.

The Omicron wave has shown the stiff consequences to safety and educational quality when school transmission is rampant. This includes school closures, use of the National Guard to fill in for sick teachers, student walkouts, talent retention problems, and high pediatric hospitalization rates with predictable consequences on mental health and education quality.

This means that limiting school-based SARS-CoV-2 transmission is important both for controlling viral evolution and providing educationally meaningful in-person learning. To formulate effective strategies to control transmission in schools, we first need to have an objective picture of the potential for schools to propagate SARS-CoV-2 spread. We discuss the limitations of current research on this topic and provide evidence of school-based transmission and its importance. Finally, we describe strategies to keep schools open, while ensuring the safety of students, staff and surrounding communities.

\section{Methodological limitations undermine findings of limited in-school transmission of SARS-CoV-2}

Early in the pandemic, four findings led to the perception of limited potential for in-school spread of SARS-CoV-2: (i) perceived reduced susceptibility of children to SARS-CoV-2 infection, (ii) apparent lower infectiousness of children, (iii) lack of identified transmission chains in schools, 
and (iv) similar rates of infection between schools and communities. However, there are significant methodological limitations associated with each of these early findings, and the ongoing pandemic has directly provided evidence that contradicts the initial findings. We detail the limitations of the evidence for these perceptions and provide strong evidence that schools can be an important SARS-CoV-2 transmission source (Table).

Susceptibility. Much of the evidence used to describe the decreased burden in children is dependent on symptom-gated testing, which is confounded by testing bias because children are more frequently asymptomatic. As a result, meta-analyses do not support the finding of reduced susceptibility to infection at this point. ${ }^{2}$ Studies with surveillance testing strategies report schoolaged children being infected at comparable levels to adults. For instance, UK prevalence surveys in October 2020 in areas with open schools found the highest prevalence of SARSCoV-2 infections in 18-25 year olds, followed by 11-18 year olds, with 5-11 year olds having comparable prevalence to working-age adults. ${ }^{3}$ This is consistent with seroprevalence survey results in Italy, Utah and Wisconsin. ${ }^{4,5}$ Notably, many studies showing lower pediatric rates were conducted in the spring 2020 when schools were closed and children had lower contact rates. ${ }^{5}$

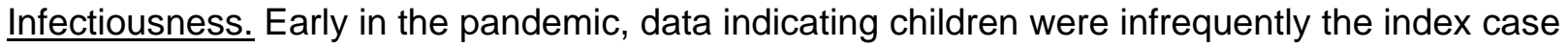
(first infection) within a family challenged the notion that children were infectious. ${ }^{6}$ Similar to disease burden estimates, this was heavily confounded by lower contact rates of children during the lockdown phase and the lower likelihood of detection of asymptomatic index cases. When children are asymptomatic, their infection may only be detected after diagnosis of an older family member, meaning the infection order does not necessarily mimic case detection. Studies based on surveillance testing find childrens' infectiousness is at least equivalent to adults. Similar infectivity between children and adults has been reported for variants of concern such as B.1.1.7. ${ }^{7}$. The trajectory of the pandemic has also undermined arguments of reduced pediatric susceptibility or infectiousness. For the week ending January 20 children accounted for 25.5\% of reported weekly COVID-19 cases (22.2\% of the US population are under 18 year old) according to the American Academy of Pediatrics website (https://www.aap.org/en/pages/2019novel-coronavirus-covid-19-infections/children-and-covid-19-state-level-data-report/).

School transmission chains. Studies have reported a lack of observed in-school transmission events between children as evidence against school-based transmission (for example ${ }^{8}$ ). This line of reasoning corresponds to the formal logical fallacy of interpreting an absence of evidence as evidence of absence. These studies relied on symptom-gated forward contact tracing, commonly used by U.S. schools. Detecting child-to-child transmission relies on the appearance and reporting of two consecutive symptomatic cases, connected by a transmission event. In a recent simulation study, we examined the performance of such an approach taking into account the low fraction of pediatric COVID-19 infections that are symptomatic (21\%) and the low likelihood of a case transmitting to others due to overdispersion (10\%). Using a Markov model of SARS-CoV-2 transmission, we found approximately $4.4 \%$ of school-based child-to-child transmission events were detectable using symptom-gated forward contact tracing. Throughout the 2020 and 2021 school years, contact tracing was frequently conducted on a voluntary basis, with the determination of the SARS-CoV-2 status of the primary contact being optional. If optouts from contact tracing were nonrandom, then we would expect contact tracing efficiency as implemented in many US schools to be lower than $4 \%$.

Community and school rates of disease. A fourth line of evidence supporting the argument that schools do not contribute to the spread of SARS-CoV-2 comes from a number of studies that show similar rates of infection in schools and their surrounding communities (for example ${ }^{9}$ ). 
With datasets incorporating multiple counties and their corresponding school district, these studies show a correlation between the county in-school infection rate and the overall county. However, this line of reasoning corresponds to the formal logical fallacy of interpreting a correlation as implying (a lack of) causality.

Ideally, we would determine the impact of school openings on the community through randomized studies. Without randomization, the causal flow could be in the opposite direction. If one starts from the premise that school transmission is more frequent than in the community and that there is free mixing between schools and communities, then the correlation between school and community COVID-19 levels would support the opposite conclusion. Chains of infection, once seeded inside schools, would spread rapidly into the surrounding communities, and communities with highest school-based transmission would also have the highest overall infection rates.

In a recent study, we simulated this scenario to ask whether the rates and kinetics of detected cases in schools and communities would let us infer that schools were responsible for transmission of SARS-CoV-2, if that were indeed the case. ${ }^{1}$ Using a Susceptible-ExposedInfectious-Recovered (SEIR) compartmental model of SARS-CoV-2 transmission in children and adults (with age-specific contact rates and location-specific transmission probabilities) we asked the question, "if transmission rates in schools were (hypothetically) far higher than the community, would detected case rates in schools allow us to see that?" Despite the simulated higher infection prevalence in children, detected case rates in children appeared similar to or lower than those of adults, and as total infection rates in the population increased, the gap between detected case rates narrowed. ${ }^{1}$ This suggests that open schools would seed chains of infection that spread rapidly into the community, and given the relatively rapid kinetics of SARSCoV-2 transmission, the infection rates in schools and communities would quickly converge. ${ }^{1}$ School reopening in the UK in the fall of 2021 led to a new spike in cases in the school-age population, which was followed shortly afterwards by a spike in cases in the 30-40 age group, consistent with a scenario where children are infected at school and subsequently infect their family members.

\section{Schools contribute to COVID-19 burden in communities}

Roughly half of the United States population is either in school, works in a school, or is a first degree contact of individuals in the previous two categories. It is not surprising that there are real-world data consistent with the possibility that in-school transmission can impact the disease burden in surrounding communities. In addition to the evidence just cited, we point out a few other key points.

Compelling evidence of the impact of in-school transmission on case counts can be found by comparing counties where schools were conducting in-person learning to areas where schools were virtual. For example, a large online study in the US reported that individuals were more likely to report COVID-19 like symptoms in areas where schools were open compared to areas with remote learning, an effect that was attenuated in communities using multiple mitigation measures. ${ }^{10}$ Similarly, school reopenings in regions of the US with limited measures in place to mitigate in-school transmission are associated with elevated case counts. ${ }^{9}$

A key concern with school reopening is the risk of superspreader events. Superspreading is an important driver of pandemics and, in particular, the COVID-19 pandemic. Ideal conditions for superspreading include prolonged indoor exposure between individuals with poor ventilation. 
Additionally, the most potent superspreaders have been individuals who are asymptomatic or presymptomatic and mobile. ${ }^{11}$ Schools combine all of these elements with clear potential consequences for the children and staff they bring together and their communities.

With that said, there is evidence that schools can operate successfully without significant inschool transmission when appropriate mitigation strategies are employed. The UK and Singapore both reopened with minimal in-school transmission in the summer term of 2020. A Japanese study matching in person and remote schooling communities showed no difference in cases in the surrounding communities between remote and in person schooling in spring 2020. ${ }^{12}$ In each of these cases, community spread in the country was extremely low (incidence was consistently less than 1/100000 in Japan). Additionally, in-person schooling was conducted with multiple layered interventions such as masking, clear ventilation guidelines, and strict hygiene practices.

\section{What can we do to open schools safely?}

Knowing that schools create an efficient setting for SARS-CoV-2 transmission, it is important that we implement multilayered strategies that are science-based and rigorously implemented. We describe several key components here.

Limit transmission by infected individuals. SARS-CoV-2 transmission is frequently airborne and aerosol-driven, even from asymptomatically-infected individuals, making it crucial to improve air quality effectively reducing indoor transmission. Appropriate ventilation is critical: regular turnover of the air inside a closed space dramatically decreases viral load. High-quality filtration units with a minimum efficiency reporting value (MERV) greater than 9 (corresponding to filters that can remove particles 3 microns or smaller in diameter) can be helpful. Ionizers purify the air in the room by creating negative ions that attach to aerosolized particles, increasing the rate at which they settle to the ground. There is a pressing need for clear science-backed standards for indoor air quality, formulated with the reduction of airborne transmission as a goal. Notably, there is a large gap between the standards that actually improve air quality and the standards that can be implemented. For example, in the US, some schools have modern HVAC with filters, but most do not, creating a problematic gap. Air quality monitoring should be conducted in schools, and parents, teachers, and school boards should be informed of the results and provided resources for needed improvements.

As a second layer of defense, high quality, well-fitting masks have been shown to provide important protection against infection. Masking policies that fail to ensure high quality masks are used effectively will undermine their efficacy. While there have been calls in the popular press for removing the requirement for masks in school, these calls are not supported by science. ${ }^{13}$ Given the waning efficacy of vaccines over time, and their vulnerability to immune evasion resulting from viral evolution, masks provide a critical layer of protection for both vaccinated and unvaccinated individuals.

Reduce the likelihood that infected individuals are in schools. Surveillance testing is a critical tool to identify and isolate infected individuals. At the university level, this has been shown to be extremely effective when testing has fast turnaround and contact tracing and isolation guidelines are clear. However slow turnaround times, using lower sensitivity rapid antigen tests, opt-in policies limiting participation and testing pools that are too large can undermine the efficacy of surveillance testing. Further, we advocate that changes to quarantine and isolation protocols should be made with the intention of limiting transmission in schools, as opposed to limiting time away from in-person learning. In addition, children who thrived under remote learning options, or 
for whom the risk of infection is too high (either due to their own health or the health of a household member) should be able to continue accessing remote learning indefinitely. This reduces the number of potentially infected individuals in school, further lowering the risk of in person schooling.

Provide community support. Even the most rigorously implemented strategies are unlikely to provide sufficient protection against the flood of cases that come from widespread community transmission. Limiting community transmission will support schools and extend the runway for safe operation. Schools might need to nimbly shift to remote learning should community burden become too high. It is critical to prepare for this possibility so that educational quality is not compromised and ensure that all students have access to the necessary technology to engage in an equitable way. This includes developing necessary technologies and investment in infrastructure to enable engagement of the most vulnerable students. It is a critically important problem to solve: we must ensure that students who are most vulnerable to the consequences of a raging pandemic and remote learning continue to receive the education they need and that their communities are safe.

Remove the fluff. We have learned that many mitigation measures are not effective and removing them will make it easier to focus on effective measures. This includes temperature checks, plexiglass barriers, and excessive surface cleaning. These mitigation measures become "hygiene theater" in that they provide false reassurance that interventions are in place while also being ineffective at preventing spread and contributing to "pandemic fatigue" declining trust in and energy for pandemic mitigations. Guidelines need to be updated and communicated as technologies are developed and science progresses. We need to move away from overly relying on vaccines to limit transmission, as they are only one part of what needs to be a multi-pronged strategy as new variants continue to emerge. While increasing levels of vaccination is an important public-health goal, as it mitigates the worst outcomes, vaccine efficacy against transmission should be complemented with other measures aimed to reduce transmission. This is particularly important as vaccine efficacy against transmission is known to wane against emerging variants and as time since vaccination increases. ${ }^{14}$ It is important that schools adopt multiple strategies to limit transmission such as masking, given a correctly-fitted N95 mask provides a critical, substantial and reliable impediment to viral transmission. ${ }^{15}$

Similarly, because some of the effective interventions (air quality improvement and testing in particular) have a nonlinear effect on risk mitigation, cutting corners on risk mitigation steps can degrade their utility very quickly. This is also hygiene theater and the use of theoretically effective but practically ineffective mitigation measures can again provide false reassurance and contribute to declining trust in mitigation. Assessing the effectiveness of these interventions in a data-driven way (for example, using CO2 monitors to assess air quality) is key. Model-based approaches should be used to pressure-test mitigation strategies, and governing authorities should update the science regularly to keep up with viral evolution.

\section{Conclusion}

Throughout the course of the pandemic, the issue of how to keep schools open safely has been a consistently contentious and complicated issue. Unfortunately, the discussion has often centered around numerous false dichotomies and limited ambition on how to truly tackle this challenge. For instance, many discussions pit ostensible direct harms to children from mitigation measures against the direct harm to children from COVID-19 infection. Other discussions pit student mental health against protection from in-person transmission of disease. Framing the question in these ways creates what appears to be an impossible dilemma. Here, we argue that 
many of these dilemmas are not really dilemmas: children are inextricably a part of the community, and the harms from in-school transmission extend to the entire community and impact the trajectory of the pandemic; schools enable disease transmission and it is possible to curb transmission; children often do better learning in person and it is possible to keep them safely in school in the midst of the pandemic with appropriate mitigations.

"Learning to live with the virus" is not synonymous with permitting rampant viral spread. Bringing the crisis phase of the current COVID-19 pandemic to an end is a goal that we all aspire towards, but it will take work on our part to make it come to pass-at present the disease is nowhere near achieving endemic conditions. The first step in that process is to limit disease spread and focusing on limiting in-school spread is critical for limiting transmission. Fortunately, we are close to the point where science-driven interventions make the goal of limiting in-school spread achievable. We should make it a public-health priority to keep schools open without accelerating the pandemic.

Table: Summary of biases and the expected impact on conclusions related to SARS-CoV2 infection in children and transmission in school settings.

\begin{tabular}{|c|c|c|}
\hline Claim & Types of bias in existing evidence & Impact of biases \\
\hline $\begin{array}{l}\text { Differential } \\
\text { susceptibility to } \\
\text { infection in children }\end{array}$ & $\begin{array}{l}\text { - Misclassification of infection status } \\
\text { due to symptom-gated testing } \\
\text { - Selection bias due to opt-in testing \& } \\
\text { reduced testing access } \\
\text { - } \quad \text { Missing link fallacy: conditional } \\
\text { probability of infection given contact } \\
\text { rate misinterpreted as probability of } \\
\text { infection }\end{array}$ & $\begin{array}{l}\text { Under-estimation of } \\
\text { susceptibility in children }\end{array}$ \\
\hline $\begin{array}{l}\text { Differential } \\
\text { infectiousness in } \\
\text { children }\end{array}$ & $\begin{array}{l}\text { - Misclassification of infection status } \\
\text { due to symptom-gated testing } \\
\text { - Misclassification of index case due to } \\
\text { asymptomatic infection \& symptom- } \\
\text { gated testing } \\
\text { - Confounding by contact rates }\end{array}$ & $\begin{array}{l}\text { Under-estimation of } \\
\text { infectiousness of children }\end{array}$ \\
\hline $\begin{array}{l}\text { Likelihood of school- } \\
\text { based transmission }\end{array}$ & $\begin{array}{l}\text { - Logical fallacy: absence of evidence } \\
\text { is not evidence of absence } \\
\text { - } \quad \text { Confounding by asymptomatic } \\
\text { infection } \\
\text { - } \quad \text { Confounding due to superspreader } \\
\text { behavior } \\
\text { - } \quad \text { Selection bias due to opt-in testing \& } \\
\text { contact tracing } \\
\text { - Misclassification due to symptom- } \\
\text { gated testing }\end{array}$ & $\begin{array}{l}\text { Under-estimation of } \\
\text { transmission chains } \\
\text { between children in } \\
\text { school settings }\end{array}$ \\
\hline $\begin{array}{l}\text { Relationship between } \\
\text { school and }\end{array}$ & $\begin{array}{l}\text { - Logical fallacy: correlation does not } \\
\text { imply (absence of) causation }\end{array}$ & $\begin{array}{l}\text { Under-estimation of } \\
\text { contribution of school }\end{array}$ \\
\hline
\end{tabular}




\begin{tabular}{|l|l|l|}
\hline $\begin{array}{l}\text { community infection } \\
\text { rates }\end{array}$ & $\begin{array}{l}\text { Red herring fallacy: no reason to } \\
\text { assume that detected case rates in } \\
\text { schools must be higher, even if } \\
\text { schools are driving transmission. } \\
\text { - } \begin{array}{l}\text { Reverse causation bias: correlated } \\
\text { rates could be due to schools driving } \\
\text { spread in communities. }\end{array}\end{array}$ & $\begin{array}{l}\text { openings to community } \\
\text { infection levels }\end{array}$ \\
& $\begin{array}{l}\text { Misclassification of infection status } \\
\text { due to symptom-gated testing } \\
\text { Confounding by asymptomatic } \\
\text { infection }\end{array}$ & \\
& & \\
\hline
\end{tabular}

\section{Author contributions}

All authors prepared and edited the manuscript.

\section{Declaration of interest}

L.F.W. reports funding from the National Institutes of Health (R35GM141821). A.C. is founder of Fractal Therapeutics.

\section{References}

1. Johnson KE, Lachmann M, Stoddard M, Pasco R, Eng M, Fox SJ, et al. Detecting in-school transmission of SARS-CoV-2 from case ratios and documented clusters. medRxiv. $2021 \mathrm{Apr}$ 28;2021.04.26.21256136.

2. Davies NG, Klepac P, Liu Y, Prem K, Jit M, Pearson CAB, et al. Age-dependent effects in the transmission and control of COVID-19 epidemics. Nature Medicine 2020 26:8. 2020 Jun 16;26(8):1205-11.

3. Flasche S, Edmunds WJ. The role of schools and school-aged children in SARS-CoV-2 transmission. The Lancet Infectious Diseases. 2021 Mar 1;21(3):298-9.

4. Barcellini L, Forlanini F, Sangiorgio A, Gambacorta G, Alberti L, Meta A, et al. Does school reopening affect SARS-CoV-2 seroprevalence among school-age children in Milan? PLOS ONE. 2021 Sep 1;16(9):e0257046.

5. Goldstein E, Lipsitch M, Cevik M. On the Effect of Age on the Transmission of SARS-CoV-2 in Households, Schools, and the Community. The Journal of infectious diseases. $2021 \mathrm{Feb}$ 1;223(3):362-9.

6. Zhu Y, Bloxham CJ, Hulme KD, Sinclair JE, Tong ZWM, Steele LE, et al. A Meta-analysis on the Role of Children in Severe Acute Respiratory Syndrome Coronavirus 2 in Household Transmission Clusters. Clinical Infectious Diseases. 2021 Jun 15;72(12):e1146-53.

7. Loenenbach A, Markus I, Lehfeld AS, an der Heiden M, Haas W, Kiegele M, et al. SARS-CoV-2 variant B.1.1.7 susceptibility and infectiousness of children and adults deduced from 
investigations of childcare centre outbreaks, Germany, 2021. Eurosurveillance. 2021 May 27;26(21):1.

8. Gillespie DL, Meyers LA, Lachmann M, Redd SC, Zenilman JM. The Experience of 2 Independent Schools With In-Person Learning During the COVID-19 Pandemic. The Journal of School Health. 2021 May 1;91(5):347.

9. Ertem Z, Schechter-Perkins EM, Oster E, van den Berg P, Epshtein I, Chaiyakunapruk N, et al. The impact of school opening model on SARS-CoV-2 community incidence and mortality. Nature Medicine 2021 27:12. 2021 Oct 27;27(12):2120-6.

10. Lessler J, Grabowski MK, Grantz KH, Badillo-Goicoechea E, Metcalf JCE, Lupton-Smith C, et al. Household COVID-19 risk and in-person schooling. Science. 2021 Jun 4;372(6546):1092-7.

11. Lewis D. Superspreading drives the COVID pandemic - and could help to tame it. Nature. 2021 Feb 1;590(7847):544-6.

12. Fukumoto K, McClean CT, Nakagawa K. No causal effect of school closures in Japan on the spread of COVID-19 in spring 2020. Nature Medicine 2021 27:12. 2021 Oct 27;27(12):2111-9.

13. Escandón K, Rasmussen AL, Bogoch II, Murray EJ, Escandón K, Popescu S V., et al. COVID-19 false dichotomies and a comprehensive review of the evidence regarding public health, COVID-19 symptomatology, SARS-CoV-2 transmission, mask wearing, and reinfection. BMC Infectious Diseases. 2021 Dec 1;21(1).

14. Buchan SA, Chung H, Brown KA, Austin PC, Fell DB, Gubbay JB, et al. Effectiveness of COVID-19 vaccines against Omicron or Delta symptomatic infection and severe outcomes. medRxiv. 2022 Jan 28;2021.12.30.21268565.

15. Andrejko KL, Pry JM, Myers JF, Fukui N, DeGuzman JL, Openshaw J, et al. Effectiveness of Face Mask or Respirator Use in Indoor Public Settings for Prevention of SARS-CoV-2 Infection California, February-December 2021. MMWR Morbidity and Mortality Weekly Report [Internet]. 2022 Feb 4 [cited 2022 Feb 6];71(6). Available from: https://www.cdc.gov/mmwr/volumes/71/wr/mm7106e1.htm 